\title{
In Vivo Validation of an Experimental Adaptive Quantitative Coronary Angiography Algorithm to Circumvent Overestimation of Small Luminal Diameters
}

\author{
David Keane, MB, MRCPI, Ed Gronenschild, PhD, Cornelis Slager, MSc, Yukio Ozaki, MD, PhD, \\ Jürgen Haase, MD, PhD, and Patrick W. Serruys, MD, PhD
}

\begin{abstract}
The reliability of quantitative coronary angiography (QCA) measurements is of fundamental importance for the study and practice of interventional cardiology. In vivo validation results have consistently reported a tendency for QCA systems to overestimate small luminal diameters. Such a systematic error may result in the underestimation of luminal gain during intracoronary procedures and in the underestimation of progression of coronary artery disease during longitudinal studies.

We report the in vivo validation results of an experimental adaptive edge-detection algorithm that was developed to reduce overestimation of small luminal diameters by incorporating a dynamic function of variable kernel size of the derivative operator and variable weighting of the first and second derivatives of the brightness profile. The results of the experimental algorithm were compared to those of the conventional parent edge detection algorithm with fixed parameters.

Dynamic adjustment of the edge-detection algorithm parameters was found to improve measurements of small $(<0.8-\mathrm{mm})$ luminal dlameters as evidenced by an intercept of $+.07 \mathrm{~mm}$ for the algorithm with variable weighting compared to $+0.21 \mathrm{~mm}$ for the parent algorithm with fixed weighting. A slope of $<1$ was found for both the parent and experimental algorithms with subsequent underestimation of large luminal dlameters.

Systematic errors in a OCA system can be identified and corrected by the execution of objective in vivo validation studies and the consequent refinement of edge-detection algorithms. The overestimation of small luminal diameters may be overcome by the incorporation of a dynamic edge-detection algorithm. Further refinements in edge-detection algorithms will be required to address the issue of underestlmation of large luminal diameters before the absolute values derived from QCA measurements can be considered accurate over the full range of clinically encountered luminal diameters.

(c) 1995 Wiley-Liss, Inc.
\end{abstract}

Key words: coronary arterlography, quantitatlve coronary angiography, validation

\section{INTRODUCTION}

Computerized quantitative coronary angiography (QCA) has fundamentally altered our approach to the assessment of interventional techniques and strategies aimed at the prevention of restenosis and progression of coronary artery disease. The reliability of QCA measurements is therefore of central importance for the validity of angiographic trials and the application of their results to clinical practice.

Overestimation of small luminal diameters by automated QCA systems has been consistently reported for both edge detection, as well as videodensitometric algorithms [1-6]. The degree of reported overestimation varies according to the individual QCA system as well as the validation model (e.g., in vitro or in vivo, focal spot size of X-ray system). In a recent multicenter validation study of QCA systems at core angiographic laboratories in
North America and Europe, we found that all 10 QCA systems validated, overestimated measurements of small luminal diameters [1]. The clinical implications of such overestimation include the reporting in clinical trials of smaller luminal gains than actually achieved at intervention, the underestimation of progression $[8,9]$ of coronary artery disease in longitudinal studies, and the underestimation of the vasospastic response to ergonovine

From the Department of Interventional Cardiology, Thoraxcenter, Erasmus University Rotterdam, and Department of Medical Informatics, University of Limburg, Maastricht, The Netherlands.

Received October 19, 1994; revision accepted February 2, 1995.

Address reprint requests to Patrick W. Serruys, M.D., Department of Interventional Cardiology, Thoraxcenter, Ee2338, Erasmus University, P.O. Box 1738, Rotterdam 3000 DR, The Netherlands. 
provocation tests [10]. Furthermore, in the analysis and interpretation of QCA validation studies, the overestimation of small luminal diameters by a given QCA system may partially compensate for the underestimation of large luminal diameters by QCA systems, resulting in the reporting of a highly favorable accuracy value for the QCA system, for the total range of diameters validated [7].

While one approach to overcome this phenomenon would be to provide a look-up-table for the results of small luminal diameter measurements based on regression analysis of in vivo validation studies, given the unpredictable degree of $\mathrm{X}$-ray scatter in the thorax and possible motion blur of the heart, a dynamic modification of the edge-detection algorithm to address this issue would be scientifically preferable. We report in vivo validation results for an experimental QCA edge detection algorithm which was developed (University of Limburg), in an attempt to overcome the problem of overestimation of small luminal diameters by incorporating an adaptive correction function. The experimental algorithm was based on a conventional parent edge detection algorithm, with fixed weighting (50:50) of the first and second derivatives. A comparable approach to algorithm modification has been reported by Sonka et al. [13], who also used an edge operator that was dependent on the approximate diameter.

To determine the efficacy of the dynamic adaptive algorithm, we compared the quantitative measurements of this experimental algorithm with those obtained from the parent algorithm with fixed weighting for the same series of in vivo angiographic stenoses. Angiographic "phantom" stenoses of known diameter, mimicking human coronary artery obstructions, were serially inserted in the coronary arteries of anesthetized pigs and the QCA measurements of the cineangiograms obtained were compared with the known dimensions of the phantoms, to determine the reliability of the two QCA algorithms.

\section{METHODS}

\section{Stenosis Phantoms}

The stenosis phantoms consisted of radiolucent acrylate or polyimide cylinders with precision-drilled eccentric circular lumens of $0.5,0.7,1.0,1.4$, and $1.9 \mathrm{~mm}$ in diameter, as previously described $[2,3]$. The outer diameters of the cylinders were 3.0 or $3.5 \mathrm{~mm}$ and all were $8.4 \mathrm{~mm}$ in length. Acrylate was used to produce the phantoms with small stenosis diameters $(0.5$ and 0.7 $\mathrm{mm}$ ), whereas the less fragile polyimide was better suited to the drilling of large stenosis diameters (1.0, 1.4 , and $1.9 \mathrm{~mm}$ ). Parallel to the stenosis lumen a second channel of $1.3 \mathrm{~mm}$ in diameter was drilled in the cylinders to attach them to the tip of 4 Fr Fogarty catheters
(Vermed, Neuilly en Thelle, France). The central lumens of these catheters contained a removable metal stilette, which was used for intracoronary insertion of the phantoms, as well as for their positioning in the radiographic isocenter during the in vivo experiments.

\section{Animal Preparation}

Following an overnight fast, four cross-bred Landrace-Yorkshire pigs of $45-50 \mathrm{~kg}$ in weight were sedated with intramuscular ketamine $(20 \mathrm{mg} / \mathrm{kg})$ and intravenous metomidate $(5 \mathrm{mg} / \mathrm{kg})$. The animals were intubated and connected to a respirator for intermittent positive-pressure ventilation with a mixture of oxygen and nitrous oxide. Ventilator settings were adjusted during the experiments to maintain the arterial $\mathrm{pH}$ at 7.35-7.45, $\mathrm{pCO}_{2}$ at $35-45 \mathrm{~mm} \mathrm{Hg}$, and the $\mathrm{pO}_{2}$ at $>150 \mathrm{mmHg}$. Anesthesia was maintained with a continuous intravenous infusion of pentobarbital $(5-20 \mathrm{mg} / \mathrm{kg} / \mathrm{hr})$.

Valved introducer sheaths (12 Fr: Vygon, Ecouen, France) were surgically placed in both carotid arteries to allow sequential insertion of the angiographic guiding catheter and the stenosis phantoms. An $8 \mathrm{Fr}$ introducer sheath was placed in a femoral artery for the introduction of a 7 Fr high-fidelity micromanometer (disposable microtip catheter, type $811 / 160$, Cordis-Sentron, Roden, The Netherlands). Jugular venous access was secured for the administration of medications and fluid. Each animal received an intravenous bolus of acetylsalicylic acid ( 500 $\mathrm{mg}$ ) and heparin (10,000 IU), and a continuous infusion of heparin $(10,000 \mathrm{IU} / \mathrm{hr})$ was maintained throughout the procedure, to prevent the formation of an intracoronary thrombus

\section{Calibration of Quantitative Coronary Measurements}

Two different calibration methods were applied to each series of quantitative coronary analysis measurements:

Conventional catheter calibration: The nontapering part of the tip of each $8 \mathrm{~F}$ polyurethane guiding catheter (El Gamal, type 4, Schneider, MN) was measured (diameters of the individual catheters ranged from 2.49 to $2.54 \mathrm{~mm}$ ) with a precision micrometer (No. 293-501, Mitutoyo, Tokyo, Japan; accuracy $0.001 \mathrm{~mm}$ ). The catheter was then introduced into the ascending aorta via the left carotid artery and engaged in the ostium of the left coronary artery. Before injecting contrast medium the catheter tip was flushed with saline and recorded on cinefilm for subsequent off-line QCA.

Calibration at the isocenter: A cylindrical metallic object (drill-bit) of known diameter $(3.0 \mathrm{~mm})$ was placed at the isocenter of the $\mathrm{X}$-ray system and recorded on cinefilm. For the two QCA systems, the available calibration procedure using automated edge detection was 
applied to the images obtained, yielding the corresponding calibration factors $(\mathrm{mm} / \mathrm{pixel})$.

\section{Coronary Angiography and Placement of Stenosis Phantoms In Vivo}

After engaging the guiding catheter in the left main coronary artery, intracoronary isosorbide-dinitrate (1 mg) was administered to control coronary vasomotor tone before the insertion of the phantoms. The stenosis phantoms were serially wedged in the left anterior descending or left circumflex artery and positioned in the radiographic isocenter using the tip of the metal wire as a marker, which was removed prior to angiography. Coronary angiography was performed by ECG (R-wave)triggered injection of $10 \mathrm{ml}$ iopamidol 370 at $37^{\circ} \mathrm{C}$ with a programmed injection rate of $10 \mathrm{ml} / \mathrm{sec}$ (rise time $=0$ ) through the $8 \mathrm{Fr}$ guiding catheter, using a pressure injector. To minimize the effect of ventilation on angiographic acquisition, the respirator was disconnected during contrast injection.

\section{Image Acquisition and Processing}

Cineangiography was performed at $25 \mathrm{frames} / \mathrm{sec}$ on a monoplane Philips Poly Diagnost C2 machine equipped with an MRC X-ray tube and powered by an Optimus CP generator (Philips Medical Systems International BV, Best, The Netherlands). The 5-inch $(12.5-\mathrm{cm})$ field mode of the image intensifier (focal spot $0.8 \mathrm{~mm}$ ) was selected, and the radiographic system settings were kept constant $(\mathrm{kVp}, \mathrm{mA}, \mathrm{msec})$ in each projection. All phantoms were imaged at the isocenter sequentially in two projections, with particular care taken to minimize foreshortening of the segment of interest and acquired on 35-mm cinefilm (CFE type 2711, Kodak, Paris, France), using an Arritechno 90 cinecamera (Arnold \& Richter, Munich, Germany) with an 85-mm lens. The cinefilms were processed by a Refinal developer (Agfa-Gavaert, Leverkusen, Germany) for $4 \mathrm{~min}$ at $28^{\circ} \mathrm{C}$. The film gradient was measured in all cases to ensure that the optical densities of interest were on the linear portion of the sensitometric curve.

From each angiogram that fulfilled the requirements of image quality for automated quantification (no superimposition of surrounding structures, no major vessel branching at the site of the phantom position), a homogeneously filled end-diastolic coronary image was selected for off-line QCA. Twenty end-diastolic frames of the phantom stenoses were suitable for edge-detection analysis. A sufficiently long luminal coronary segment was selected for quantitative analysis on all images.

\section{Automated Edge Detection Algorithms}

The software for both the experimental and the conventional parent edge detection algorithms were loaded on the same QCA hardware components $[2,11,15]$. On both the conventional and experimental QCA system, a $6.9 \times 6.9 \mathrm{~mm}$ region of interest within the $18 \times 24-\mathrm{mm}$ cineframe is digitized into a $512 \times 512$-pixel matrix using a CCD-camera ( 8 bits $=256$ density levels) resulting in a final resolution of $1,329 \times 1,772$ pixels. A correction for pincushion distortion [14] was applied for both series of analyses. For both systems, the user defines a number of centerline points within the arterial segment which are subsequently connected by straight lines, serving as a first approximation of the vessel centerline. The edge-detection algorithm is carried out in two iterations. First, the model is the initially defined centerline; second, the model is a recomputed centerline, determined automatically as the midline of the contour positions, which were detected in the first iteration.

The basic automated edge-detection algorithms for both the experimental and conventional parent algorithms are similar and are based on the first and second derivative functions applied to the brightness profiles along scanlines perpendicular to a model, using minimal cost criteria. For the conventional system, the weighting of the first and second derivatives is fixed at 50:50 for analysis of all luminal diameters. For the experimental algorithm, the following approach was adopted. A number of computer simulations were performed to gain insight into the parameters responsible for the overestimation at small vessel sizes. Theoretical density profiles of circular lumens were convolved with a gaussian function to reflect the X-ray system's blurring function. On these profiles, the edge detection algorithm was applied. It was observed that the computed diameters are larger than the true diameters below $1.5 \mathrm{~mm}$. The overestimation increased with pixel size, the width of the blurring function, and the weight factor of the second derivative $[16,17]$. Also, the smaller the diameter, the larger the overestimation. On the other hand, a small kernel size of the derivative operator has a favorable effect on the results because it compensates for the limited number of pixels defining the extent of small diameters [15]. Note, however, that small kernel size makes the edge-detection algorithm more sensitive to noise in the image, as fewer pixels are involved in the computation. On the basis of these simulations, an algorithm was generated which adaptively varies the weighting of the first and second derivative and adjusts the kernel size as a function of the approximate diameter measured in the first iteration. For small diameters, the ratio of the first to the second derivative was proportionally greater. Moreover, the kernel size of the derivative operator was adjusted to compensate for the limited number of pixels defining the extent of small diameters $[15,16]$. The experimental algorithm was adaptive only up to (and attenuated toward) a first iteration diameter measurement of $1.7 \mathrm{~mm}$. 


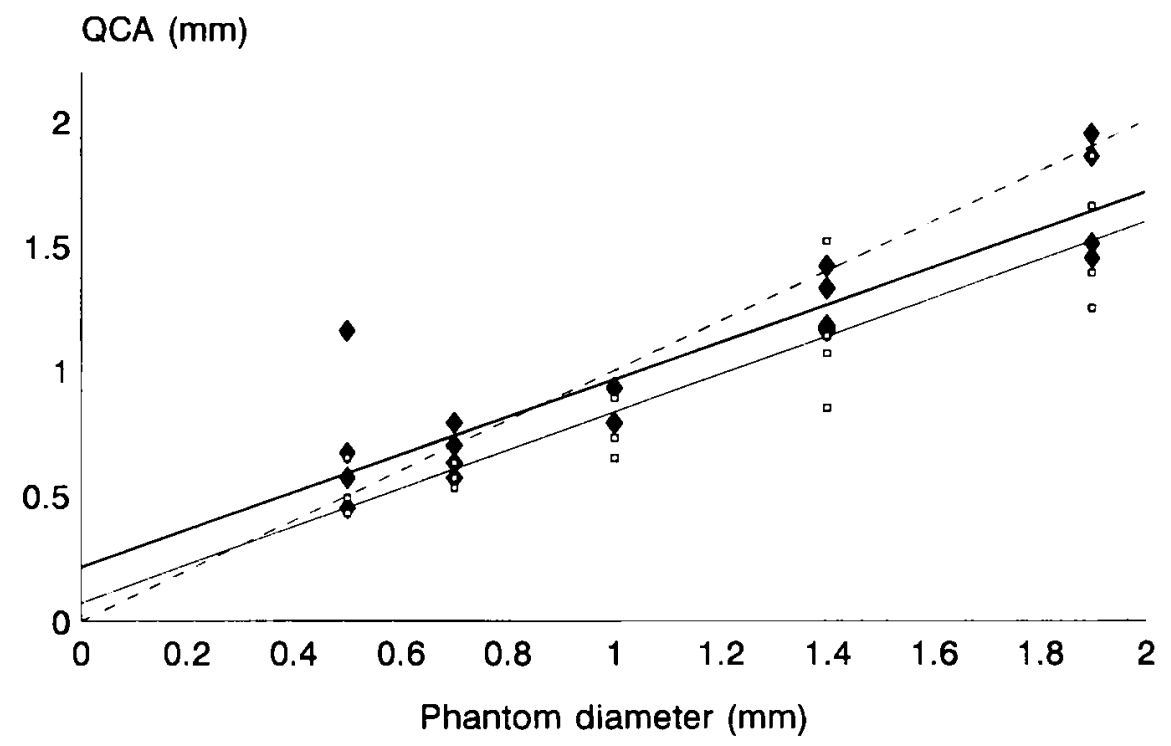

Fig. 1. QCA measurements calibrated by the angiographic catheter of the experimental and conventional edge-detection algorithms were plotted against the true values of the stenosis channels by linear regression. The results of the experimental algorithm are given as light squares and a light continuous regression line; the results of the conventional algorithm are

\section{Measurement of the Obstruction Diameter}

Once the contours of the stenosis phantoms were defined, the diameters of the artificial obstruction were automatically derived from the diameter function on each coronary analysis system. For the purpose of comparative validation, user interaction on the computerized reconstruction of phantom contours was excluded.

\section{Statistical Analysis}

Using both calibration methods (calibration at the isocenter and catheter calibration), the individual measurements for obstruction diameter obtained by the two QCA algorithms were compared with the true phantom diameters. The mean of the signed differences between the measurement values ("obstruction diameter") and the true diameters of the phantom stenoses was considered an index of accuracy and the standard deviation of the differences an index of precision. The mean absolute error was derived from the mean of the absolute (unsigned) differences between the measured values and the true diameters The measured values were plotted against the true phantom diameters by linear regression. Measurements with the same algorithm of the same stenoses derived from calibration by the catheter and calibration at the isocenter were compared by Student's $t$-test for paired data. given as dark diamonds and a dark continuous regression line; and the line of identity is represented by the interrupted line. Overestimation of small luminal diameters as seen with the conventional aigorithm [y $=0.21 \mathrm{~mm}+0.75(x)]$ was avoided by the experimental algorithm by the incorporation of adaptive dynamic functions $[y=0.07 \mathrm{~mm}+0.76(x)]$.

\section{RESULTS}

The experimental algorithm with variable kernel size and variable weighting of the first and second derivative was found to be effective in the reduction of overestimation of QCA measurements of small luminal diameters. This improvement in the performance of QCA measurements of small luminal diameter is evidenced in Figures 1 and 2 , where the results for the experimental algorithm are compared to those of the parent algorithm both when the measurements were calibrated by using the catheter as a scaling device (Fig. 1) and when measurements were calibrated at the isocenter (Fig. 2). The values of the linear regression analysis are presented in Table I.

When QCA measurements were calibrated by the catheter, the conventional edge-detection algorithm yielded an intercept of $+0.21 \mathrm{~mm}$ and a slope of $0.75(x)$. This overestimation of small diameters was reduced by the experimental algorithm, which had an intercept of $0.07 \mathrm{~mm}$ and a slope of $0.76(x)$.

Calibration of QCA measurements at the isocenter for the conventional edge-detection algorithm, resulted in both a greater positive shift (intercept) and a higher slope of $+0.30 \mathrm{~mm}$ and $0.80(\mathrm{x})$, respectively. This overestimation of small diameters was reduced by the experimental algorithm, which had an intercept of $0.05 \mathrm{~mm}$ and a slope of $0.88(\mathrm{x})$.

Given the degree of overestimation of small diameters 


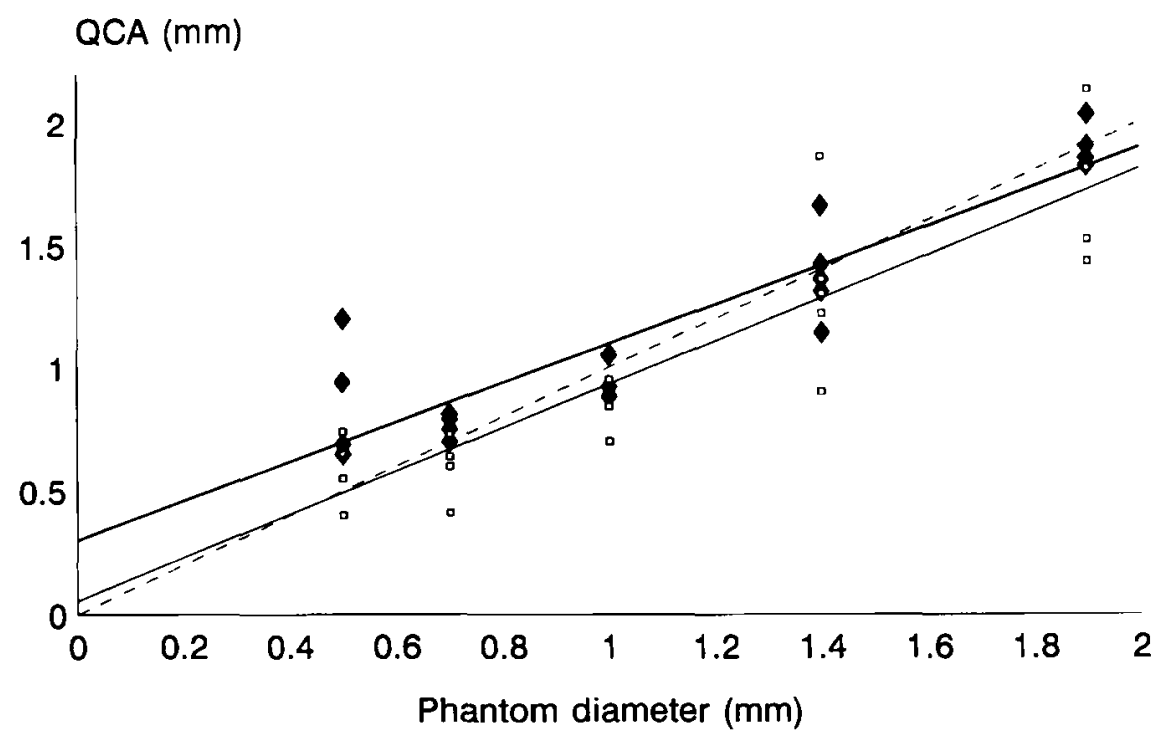

Fig. 2. QCA measurements calibrated at the isocenter of the experimental and conventional edge-detection algorithms were plotted against the true values of the stenosis channels by linear regression. The results of the experimental algorithm are given as light squares and a light continuous regression line; the results of the conventional algorithm are given as dark di- amonds and a dark continuous regression line; and the line of identity is represented by the interrupted line. Overestimation of small luminal diameters as seen with the conventional algorithm [y $=0.30 \mathrm{~mm}+0.80(x)]$ was avolded by the experimental algorithm by the incorporation of adaptive dynamic functions $[y=0.05 \mathrm{~mm}+0.88(x)]$.

TABLE I. Luminal Diameter QCA Measurements Calibrated by Catheter or by Isocenter for Experimental and Conventional QCA Algorithms*

\begin{tabular}{lcccccc}
\hline & Accuracy $(\mathrm{mm})$ & Precision $(\mathrm{mm})$ & Mean error $(\mathrm{mm})$ & Correlation & SE (mm) & Regression (mm) \\
\hline Conventional QCA catheter calibration & -0.06 & \pm 0.23 & 0.17 & 0.89 & \pm 0.20 & $0.21+0.75(\mathrm{x})$ \\
Experimental QCA catheter calibration & -0.21 & \pm 0.22 & 0.23 & 0.91 & \pm 0.19 & $0.07+0.76(\mathrm{x})$ \\
Conventional QCA isocenter calibration & 0.07 & \pm 0.21 & 0.15 & 0.91 & \pm 0.19 & $0.30+0.80(\mathrm{x})$ \\
Experimental QCA isocenter calibration & -0.08 & \pm 0.24 & 0.20 & 0.89 & \pm 0.24 & $0.05+0.88(\mathrm{x})$ \\
\hline
\end{tabular}

*Note the lower intercept values for the regression line of the experimental algorithm which incorporates variable kernel size of the derivative operator and variable weighting of the first and second derivative of the brightness profile

by the conventional system (particularly during calibration at the isocenter) in conjunction with the underestimation of larger diameters, it is not surprising that the overall accuracy (mean of the signed differences) for the validated range of $0.5-1.9 \mathrm{~mm}$ was more favorable for the conventional algorithm on account of the compensation [7] of the positive (small-diameter) and negative (large-diameter) signed values. Furthermore, despite the low slope of the conventional algorithm $(0.75(x)$ and $0.80(x)$ for the catheter and isocenter calibration, respectively), the mean error [mean of the absolute (unsigned) differences between the measured values and the true phantom values] for the conventional algorithm $(0.17$ and $0.15 \mathrm{~mm}$ ) was lower than for the experimental algorithm $(0.23$ and $0.20 \mathrm{~mm})$, on account of the greater positive shift associated with the conventional algorithm and associated superior performance in larger luminal diameters.

\section{DISCUSSION}

The key findings of this study were (1) validation by the analysis of in vivo stenoses of a standard conventional QCA algorithm reveals a well-recognised problem of overestimation of small luminal diameters as well as underestimation of larger luminal diameters; (2) application of an experimental dynamic algorithm with adaptive weighting of the first and second derivative and an adjustive kernel size to circumvent the problem of overestimation of small luminal diameters results in a reduction in such overestimation of small $(<0.8-\mathrm{mm})$ luminal diameters; (3) application of this experimental adaptive algorithm to larger luminal dimensions $(>0.8 \mathrm{~mm}) \mathrm{re}-$ sults in greater underestimation of measurements, particularly when calibrated by the catheter; and (4) although the reduction in measured diameter achieved by application of the adaptive algorithm is greatest for small lumi- 
nal diameters and attenuated for larger diameters, the experimental algorithm in its current form (the algorithm is currently programmed to exert some activity up to diameters of $1.7 \mathrm{~mm}$ ) confers no overall benefit for the whole range of $0.5-1.9 \mathrm{~mm}$ diameters validated on account of a greater underestimation of vessels $>0.8 \mathrm{~mm}$.

\section{Overestimation of Small Luminal Diameters}

Overestimation of small luminal diameters is a frequent finding of in vivo validation studies [1] and effective measures attempting to increase our understanding and to overcome such overestimation are required. The clinical implications of such overestimation of small luminal dimensions are significant. If in a clinical trial using the conventional algorithm, an improvement in coronary luminal diameter is achieved by balloon angioplasty or rotablator from $0.4 \mathrm{~mm}$ preprocedure to 1.8 mm postprocedure, a gain of 1.05 or $1.12 \mathrm{~mm}$ for catheter or isocentric calibration, respectively, will be attributed to the device, instead of a true luminal gain of 1.40 $\mathrm{mm}$. Similarly, progression of a lesion in a longitudinal study from a minimal luminal diameter of $1.0-0.4 \mathrm{~mm}$ would be underestimated by the conventional algorithm (progression would be estimated to be 0.45 or $0.48 \mathrm{~mm}$ for catheter or isocentric calibration, respectively). In the current form of the experimental adaptive algorithm with variable weighting programmed up to a diameter of 1.7 $\mathrm{mm}$, no significant benefit would be conveyed over the conventional algorithm-the luminal gain as above would be reported as 1.06 or $1.23 \mathrm{~mm}$ for catheter or isocentric calibration, respectively, and the progression as above would be reported as 0.46 or 0.53 for catheter or isocentric calibration, respectively.

The reasons for the overestimation of small luminal diameters by QCA systems remain unclear; potential factors include a point spread function relating to the focal spot size and limited resolution of the entire X-ray imaging chain [16] and too large a pixel size relative to the vessel diameter, as well as too great a kernel size of the derivative operator. While corrective numerical functions, based on the known pattern of underestimation or overestimation of QCA measurements, could be applied to the results of angiographic trials, it may be more effective and reliable to correct the systematic inaccuracies of a QCA system by the development and fine-tuning of dynamic adaptive algorithms.

We have demonstrated that the overestimation of luminal diameters of $<0.8 \mathrm{~mm}$ can be effectively reduced by the incorporation of a dynamic function of variable kernel size of the derivative operator and variable weighting of the first and second derivative in the edge detection algorithm. The experimental edge detection algorithm, however, will require further modification to address the low slope of the system; i.e., the underesti- mation of larger luminal diameters before it could be adopted in clinical practice.

\section{Calibration}

The use of angiographic catheters for the calibration of quantitative coronary analysis systems may influence the outcome of luminal diameter measurements. Variations in catheter composition may result in varying $\mathrm{X}$-ray attenuation [18-20] and therefore in differences in the automated detection of the contour points. In our study, only one type of catheter was used for calibration; therefore, the influence of different materials on calibration was excluded. The results of the present study show that the values using catheter calibration are smaller than those using calibration at the isocenter $(P<0.001)$. Theoretically, a greater distance between image intensifier and catheter tip than between image intensifier and isocenter would result in out-of-plane magnification producing smaller calibration factors $(\mathrm{mm} / \mathrm{pixel})$. This error can be circumvented by out-of-plane correction as proposed by Wollschläger et al. [21] or by calibration at the isocenter of the $X$-ray system.

\section{QCA Validation Studies}

Given the increasing role of QCA in the evaluation of interventional cardiology [7], it has become essential that the performance of QCA itself should undergo objective and scientific evaluation. While in vitro test series are more easily standardized, their results are not always representative of QCA measurements of clinical angiograms, as heterogeneous beam scattering and variable bacground density within the thorax and the potential influence of motion blur are unpredictable. In vivo validation studies on the other hand are of clinical relevance and provide an indication of the reliability of clinical angiographic trials, the significance of their failure to detect relative changes in luminal diameter and how much importance should be attributed to absolute values of luminal diameter derived from individual QCA systems. Furthermore, it is only by detailed validation studies that systematic errors in QCA systems can be identified and thereby provide guidance for the refinement of algorithms incorporated within QCA software.

\section{Statistical Parameters for QCA Studies}

Investigators reporting and readers interpreting QCA data should be familiar with the limitations of the conventional statistical parameters used to described QCA validation studies [7]. As recently described in detail [7], the reporting of the "accuracy" of QCA measurements over a validated range may fail to convey the error to be expected from a given QCA measurement on account of cancellation of the signed differences of overestimated (below the intersection of the regression and identity 
lines) and underestimated (above the intersection of the regression and identity lines) measurements. This point may be revealed by the reporting of the absolute mean error, which is independent of the sign $(+/-)$ of the differences between the true and measured values-it can be seen in the results of our study that the absolute mean error was consistently greater in value than the value of the accuracy for both the conventional and experimental algorithms (Table I). Alternatively, the reader might be well served by the provision of the accuracy of measurements at a number of different dimensions, rather than for the entire validated range as reported conventionally; review of Figure 2 clearly shows that the accuracy of measurements for dimensions of $0.4-0.8$ $\mathrm{mm}$ is superior with the experimental algorithm, that the accuracy for dimensions of $1.0-1.2 \mathrm{~mm}$ is equivalent for the experimental and conventional algorithms, and for dimensions of 1.4-1.9 mm that the accuracy of measurements with the conventional algorithm is superior to those of the experimental algorithm in its current form. For the same reason, precision of QCA measurements will be dependent on the range of validated diameters, while the standard error of the estimate (SEE) will more closely reflect the random error or noise to be expected from the QCA system particularly after correction for an intercept of 0 and a slope of 1 (SEEc) $[4,7,22]$.

\section{CONCLUSION}

Systematic errors in a QCA system can be identified and corrected by the execution of objective in vivo validation studies and the consequent refinement of edgedetection algorithms. The overestimation of small luminal diameters by QCA measurements may be overcome by the incorporation of an iterative algorithm with dynamic adjustment of the kernel size of the derivative operator and adaptive weighting of the first and second derivative of the brightness profile of the vessel in relation to the diameter to be detected. Further refinements in edge-detection algorithms will be required to address the issue of underestimation of large luminal diameters before the absolute values derived from QCA measurements can be considered to be accurate over the full range of clinically encountered luminal diameters.

\section{ACKNOWLEDGMENT}

Dr. D. Keane is a recipient of a travel grant from the Peel Medical Research Trust, London, U.K.

\section{REFERENCES}

1. Keane D, Haase J, Slager C, Montauban van Swijndregt E, Lehmann K, Ozaki Y, DiMario C, Kirkeeide R, Serruys PW: Com- parative validation of quantitative coronary angiography systems: Results and implications from a multicenter study using a standardized approach. Circulation (in press), 1995.

2. Haase J, Di Mario C, Slager C, van der Giessen W, den Boer A, de Feyter PJ, Reiber JHC, Verdouw PD, Serruys PW: In-vivo validation of on-line and off-line geometric coronary measurements using insertion of stenosis phantoms in porcine coronary arteries. Cathet Cardiovasc Diagn 27:16-27, 1992.

3. Haase J, Linden $M$ van der, Di Mario C. Giessen $W$ van der, Foley D, Serruys PW: Can the same edge-detection algorithm be applied to on-line and off-line analysis systems? Validation of a new cinefilm-based geometric coronary measurement software. Am Heart J 126:312-321, 1993.

4. Haase J, Keane D, Di Mario C, Escanned J, Slager C, Serruys PW: How reliable are geometric coronary measurements? In vitro and in vivo validation of digital and cinefilm-based quantitative coronary analysis systems. In Serruys PW. Foley DP, de Feyter PJ, (eds): "Quantitative Coronary Angiography in Clinical Practice." Dordrecht: Kluwer Academic Publishers, 1994, pp 27-50.

5. Beauman GJ, Reiber JHC, Koning H, van Houdt RCM, Vogel RA: Angiographic core laboratory analyses of arterial phantom images: Comparative evaluations of accuracy and precision. In Reiber JHC, Serruys PW (eds): "Progress in Quantitative Coronary Angiography." Dordrecht: Kluwer Academic Publishers, 1994, pp 87-104.

6. DiMario C, Haase J, den Boer A. Serruys PW: Edge detection versus densitometry for assessing stenosis phantoms quantitatively: An in-vivo comparison of in porcine coronary arteries. Am Hean J 124:1181, 1992.

7. Keane D, Serruys PW: Quantitative coronary angiography: An integral component of interventional cardiology. In Topol EJ, Serruys PW (eds): "Current Review of Interventional Cardiology." 2nd Ed. Philadelphia: Current Medicine, 1995 (in press).

8. De Feyter PJ, Seruys PW, Davies MJ, Richardson P, Lubsen J, Oliver MF: Quantitative coronary angiography to measure progression and regression of coronary atherosclerosis: Value, limitations, and implications for clinical trials. Circulation 84:412423,1991

9. Haase J, Slager C, Keane D, Foley D, den Boer A, Doriot P. Serruys PW: Quantification of intracoronary volume by videodensitometry: A validation study using fluid filling of human coronary casts. Cathet Cardiovasc Diagn 33:89-94. 1994.

10. Ozaki Y, Keane D, Haase J, Baptista J, Meneveau N, de Feyter P, Takatsu F, Serruys PW: Temporal variability and correlation with geometric parameters in vasospastic angina: A quantitative angiographic study. Eur Heart J 15:61-67, 1994.

11. Reiber JHC, Booman F, Tan HS, Slager CJ, Schuurbiers JHC, Gerbrands JJ, Meester GT: A cardiac image analysis system: Objective quantitative processing of angiocardiograms. Comput Cardiol 239-242, 1978.

12. Serruys PW, Booman F, Troost GJ, Reiber JHC, Gerbrands JJ, van den Brand $M$, Cherrier F, Hugenholtz PG: Computerized quantitative coronary angiography applied to percutaneous transluminal coronary angioplasty: Advantages and limitations. In Kaltenbach M, Gruntzig A. Rentrop K, Bussmann WD (eds): "Transluminal Coronary Angiography and Intracoronary Thrombolysis. Coronary Heart Disease." Vol. IV. Berlin: SpringerVerlag, 1982, pp 110-124.

13. Sonka M, Reddy GK, Winniford MD, Collins SM: Adaptive simultaneous coronary border detection: A method for accurate analysis of small diameter vessels. Comput Cardiol 109-112, 1993.

14. Gronenschild E, Janssen J, Tijdens F: CAAS II: A second gen- 
eration system for off-line and on-line quantitative coronary angiography. Cathet Cardiovasc Diagn (in press), 1994.

15. Linderer T, Wunderlich W, Bachs B, Noering J, Schroeder R: Edge detection in quantitative coronary angiography (QCA): The impact of image zoom on edge positioning, accuracy, precision and discrimination. Comput Cardiol 99-102, 1992.

16. Beier J, Oswald H, Fleck E: Edge detection for coronary angiograms - error correction and impact of derivatives. Comp Cardiol 513-516, 1992.

17. Herrington DM, Siebes M, Walford GD: Sources of error in quantitative coronary angiography. Cathet Cardiovasc Diagn 29:314321, 1993.

18. Reiber JHC, Kooijman CJ, Den Boer A, Serruys PW: Assessment of dimensions and image quality of coronary contrast catheters from cineangiograms. Cathet Cardiovasc Diagn 11:521-531, 1985.
19. Fortin DF, Spero LA, Cusma JT, Santoro L, Burgess R, Bashore TM: Pitfalls in the determination of absolute dimensions using angiographic catheters as calibration devices in quantitative angiography. Am J Cardiol 68:1176-1182, 1991.

20. Herrman J, Keane D, den Boer A, Sermys PW: Radiological quality of coronary guiding catheters: A quantitative analysis. Cathet Cardiovasc Diagn (in press), 1994.

21. Wollschläger $H$, Zeiher AM, Lee $P$, Solzbach U, Bonzel T, Just $H$ : Optimal biplane imaging of coronary segments with computed triple orthogonal projections. In Reiber JHC, Serruys PW (eds): "New Developments in Quantitative Coronary Arteriography." 1st Ed. Dordrecht: Kluwer Academic Publishers, 1983, pp 1321.

22. Keane D, Serruys PW: Quantitative coronary angiography: Current limitations and practical guidelines. Am Coll Cardiol Curr J Rev 3:50-53. 1994. 